\title{
Geo-Based Mixed Reality Gaming Market Analysis
}

\author{
Chutisant Kerdvibulvech \\ Graduate School of Communication Arts and Management Innovation, National Institute of Development Administration, 148 Seri \\ Thai Rd., Klong-Chan, Bangkapi, Bangkok 10240, Thailand
}

Correspondence should be addressed to Chutisant Kerdvibulvech; chutisant.ker@nida.ac.th

Received 28 October 2021; Accepted 31 December 2021; Published 17 February 2022

Academic Editor: Zheng Yan

Copyright (c) 2022 Chutisant Kerdvibulvech. This is an open access article distributed under the Creative Commons Attribution License, which permits unrestricted use, distribution, and reproduction in any medium, provided the original work is properly cited.

\begin{abstract}
Mixed reality has constantly been a symbol of the future for many years because it integrates many related areas of art and science to produce superior interactive experiences. Recently, it has become tremendously popular with location-based features that allow a new immersive interaction between people. In this paper, we present a novel research of pioneering geo-based mixed reality games, including our own research, to explore the evolution of mixed reality games using location-based features. Five major geo-based mixed reality games are selected for analysis based on the periods built: Human Pacman, AR Car Game, Ingress, Pokémon Go, and AR Mario Kart Live. The geo-based mixed reality gaming market analysis is explored extensively using the semiqualitative method to discover the relationships between the independent variable (year built) and the dependent variables (number of academic paper downloads, Google Trends, revenues, and number of downloads). We then compare each game based on the year built, the devices used in the game, the game purposes, the number of academic paper downloads, the revenues, the number of downloads, and the types of virtual items in the game, to deeply understand the evolution of geobased mixed reality games. Finally, recommendations for future directions are included.
\end{abstract}

\section{Introduction}

As the world has been technologically experiencing an extraordinary rate of change, especially during the COVID-19 era [1], mixed reality (MR) has become very popular in the related fields of computer vision, artificial intelligence, and digital marketing. Since it is a fascinating experience of a real-world environment mixed, enhanced, and/or integrated by graphically virtual scene, it is a great tool to change movie theatres and existing 3D technology into 4D and beyond. More widely, it can be used in many applications in various industries such as entertainment, digital marketing, and tourism. It has thoroughly become a trending topic both in research laboratories and in commercial sectors worldwide. More recently, because of advancements in smartphones with high-speed internet, this area has been even more extremely and quickly popular when using geo-based features for creating new mixed reality games [2]. From the research perspective, the word "mixed reality" has a broad spectrum that includes "augmented real- ity" as well. Hence, this paper will use the term mixed reality all along the paper.

This paper is aimed at presenting a novel outline of pioneering geo-based mixed reality games, including our own research [3], for the first two decades of the twenty-first century. We particularly focus on exploring five significant games which are Human Pacman, AR Car Game, Ingress, Pokémon Go, and AR Mario Kart Live. We first provide a full explanation of each game and how it works. After that, we propose our geo-based mixed reality gaming market analysis from each geo-based mixed reality game in detail. Each game is compared and analyzed based on the significant details of the games. Critical factors for future works of geo-based mixed reality games are suggested. This paper is organized as follows. Section 1 in this paper is devoted to the general introduction of mixed reality. The paper is then divided into two parts: an explanation of each selected geo-based mixed reality game (Section 2), methodology (Section 3), and geo-based mixed reality gaming market analysis (Section 4). Section 2 gives the outline of five 
selected geo-based mixed reality games and their progress in the last two decades. Next, Section 3 in this paper discusses our research methodology. Then, Section 4 presents the geo-based mixed reality gaming market analysis for each geo-based mixed reality game. Finally, Section 5 concludes the paper. We also forecast the factors and the possible future directions for this geo-based mixed reality field.

\section{Geo-Based Mixed Reality Games}

Five pioneering location-based mixed reality games in the past two decades, including our own research, will be discussed in this section. To begin with, one of the very first research works for geo-based mixed reality games which have received great popularity is Human Pacman presented in 2004 [4]. As shown in Figure 1, Cheok et al. invented this geo-based mixed reality game using the idea of Pac-Man, a traditional maze arcade game, by creating virtual cookies which can be overlaid on the real scene. Their research aim is to integrate the computer gaming experience immersively with mobile and human-social gaming. By using sensor fusion and global positioning system receiver to track positions and locations of players, in this game, players can touch virtual cookies and get real-time interactive experiences with seamless transitions between the real world and virtual world. This research has gained great popularity among the world's famous television media which are featured in CNN news, Discovery Channel, and TED Talks. It is important to note that this work is mainly for research purposes in the universities, conducted mainly in Nanyang Technological University and National University of Singapore.

Second, a pioneering form of real remote-controlled car gaming with the scoring feature for geo-based mixed reality games is our own research work, shortly called AR Car Game presented in 2010. The full name is Augmented Reality Car Game. As illustrated in Figure 2, Kerdvibulvech [5] built an interactive geo-based game for detecting a real remote-controlled car using computer vision in a gamified way. Our game estimates the location of the real remotecontrolled car using the yellow information of the image, and then, virtual items are overlaid on the real scene so that a user can command a car to collect and capture the virtual items interactively in real time. Virtual items used in this game include mushrooms and coins. Also, in the game, it has a unique score for each virtual item. For instance, when the mushroom is captured, a user gains two points. When the coin is captured, a user will receive only one point. In this way, every user should attempt to control a remotecontrolled car to collect and capture virtual items which give the top points as quickly as possible. Most importantly, this geo-based game can give each user some feedbacks and the evaluation score interactively based on the car control skills. For example, every user is automatically classified into three different levels: an expert, an intermediate driver, and a beginner. At that time, the mixed reality game for detecting a small physical remote-controlled toy with the evaluation scoring feature, we believe, was expected to be very new for a real remote-controlled toy or car gaming interactively. By

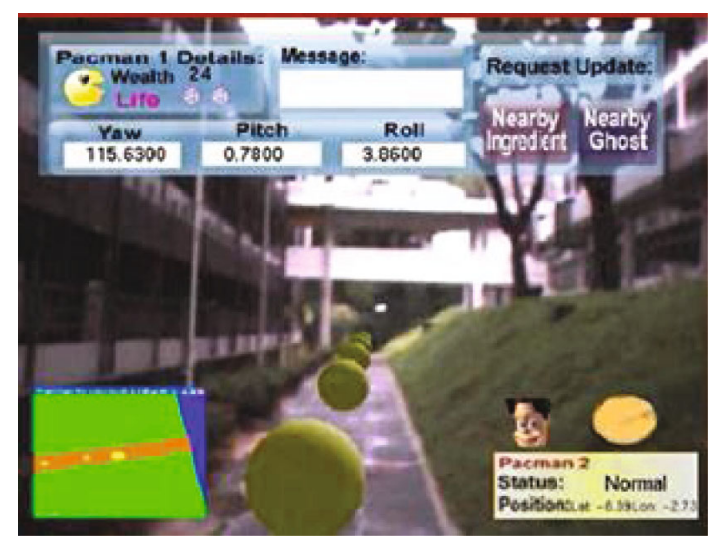

Figure 1: Human Pacman, the geo-based mixed reality game using the concept of a traditional maze arcade game [4].

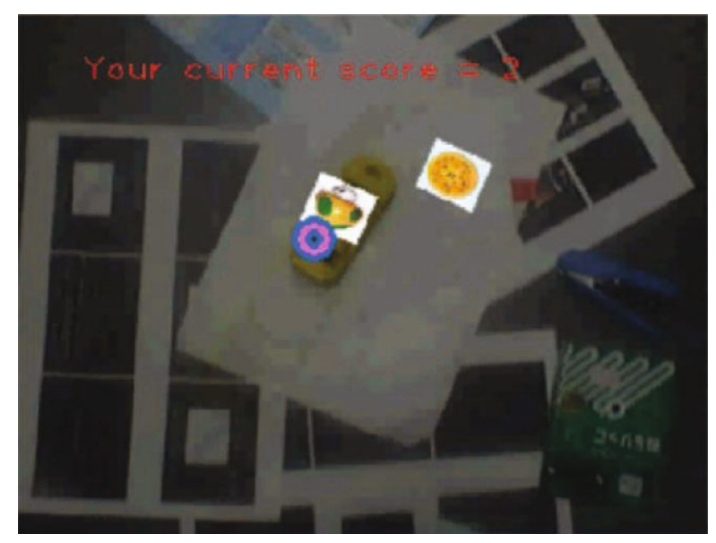

FIgURe 2: AR Car Game, a physical remote-controlled car game with the scoring feature using geo-based mixed reality [5].

the way, it is interesting to note that this work is aimed for research purposes in the university, similar to the work of Human Pacman.

Third, three years later in 2013, Niantic Inc. created a freemium geo-based mixed reality mobile game, called Ingress, as depicted in Figure 3. Similar to Human Pacman, a global positioning system is used to locate portals, while these portals are in closeness to the location of the players in the physical world using their smartphone in real time. This game is later called Ingress Prime by many players for a revamped version of the conventional game. Although this game is for commercial purposes which are different from the two aforementioned research-based games, it is a freeto-play game available for download in both iOS and Android operating systems. Although this game reached sufficient commercial success to sustain the game, their success seems to be relatively small when compared to the next game from their company that makes use of the world's famous Pokémon characters and introduces the groundbreaking geo-based mixed reality game.

Fourth, it is very obvious that, in 2016, Pokémon Go is the worldwide most popular mobile game and geo-based mixed reality technology is its main unique contribution. Niantic Inc. built this geo-based mixed reality game using data from Ingress to populate the locations for adding up 


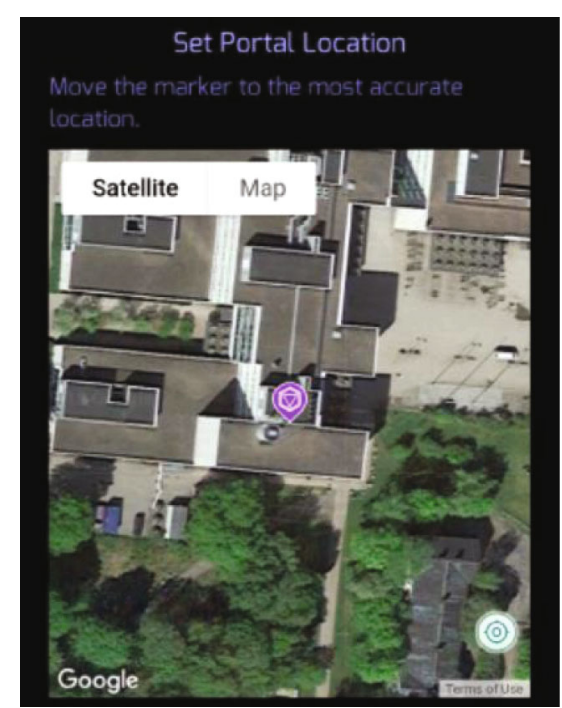

FIgURe 3: Portal submission from Niantic's Ingress, a freemium geo-based mixed reality mobile game in real-world map data [6].

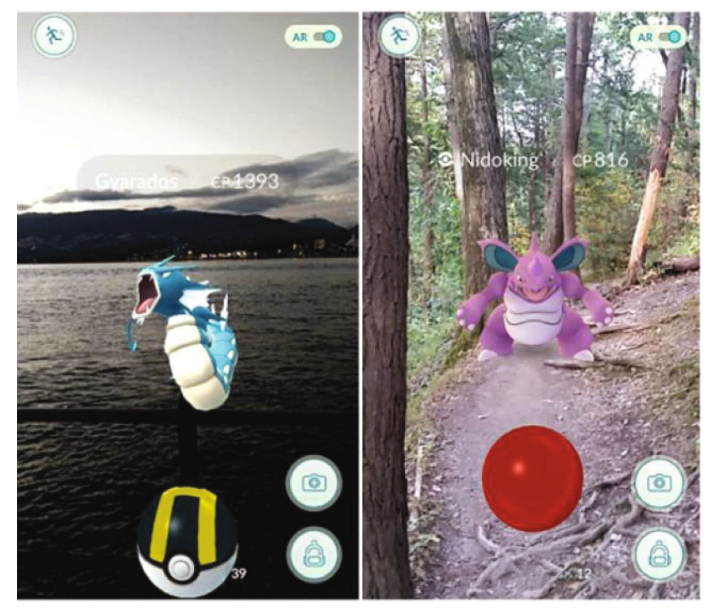

Figure 4: Niantic's Pokémon Go, the groundbreaking geo-based mixed reality game using data from Ingress to populate the locations [11].

the game components in real time [7], such as Gyms and PokéStops which are the physical world landmarks in places, such as universities, historical monuments, department stores, temples, shrines, and spirit houses. Virtual creatures are called Pokémon in this game. In the first release of Pokémon Go, each player is supposed to physically walk/run to several locations to face these virtual Pokémons, fight them with virtual Poké balls, and catch them to the player's collection. Figure 4 illustrates some examples of the game in different locations. Again, similar to Human Pacman in 2004 and Ingress in 2013, a global positioning system is used to realistically put each Pokémon onto the actual world map. A Pokémon will only appear in the game for any player whose location and position in the real world are in predefined proximity with that Pokémon. Also, similar to AR Car Game in 2010, Pokémon Go allows the players to capture virtual items (i.e., virtual Pokémons) which appear as if they are in the same location in the physical world and give them feedback and the level of each player interactively based on the skills in a fascinating way. But rather than three different levels as in AR Car Game, each player of Pokémon Go has up to 40 different levels at first and this level cap remains for several years [8]. In 2021, this level cap is increased to 50 different levels allowing players to challenge new tasks to achieve the higher level. It is important to note that this game is mainly for commercial purposes. Even though it is free to download and play, there is the incentive to spend real money on the game [9], such as buying XP boosters, PokeCoins, Poké balls, Raid Passes, special event tickets, avatar's styling accessories, and avatar's special postures. For example, the players can spend money for purchasing Poké balls in the game which have different rates and pay via credit card. Each rate depends on the type and capacity of Poké balls differently. With their lovely characters of Pokémon, the unique features of geo-based mixed reality, and the continuous popularity/support from the strong player communities, all of these make Pokémon Go, by far, one of the most profitable mobile games on earth [10]. In terms of profit in 2021, this game grossed enormously more than five billion USD in lifetime revenue in just five years. Since Niantic has been continuously releasing many updates between 2020 and 2021 to cope with the situations of work from home and social distancing, it is essential to note that the weekly player spending has surprisingly increased by approximately 70\% amid the COVID-19 pandemic, even though this outbreak has tremendously impacted and devastated so many small and medium businesses in various countries around the world.

Fifth, in late 2020, Nintendo invented AR Mario Kart Live: Home Circuit, which is similar to our work of AR Car Game (2010) in terms of using mixed reality for real remote-controlled car games and the story and characters of fictional mascots. As shown in Figure 5, by using the mixed reality hardware, AR Mario Kart Live: Home Circuit allows players to race fictional mascots, such as Mario and Luigi, at their own home through immersive experiences virtually. It consists of a real remote-controlled car that is installed with a single camera for capturing the real scene in-game, while obstacles and opponents are built virtually to make the game more entertaining. Therefore, it allows the players to design racetracks and race as fictional mascots across the car race track virtually. After the game was released, it has sold more than one million copies worldwide and been nominated at The Game Awards 2020 for the category and type of Best Family. By the way, note that this game is mainly for commercial purposes.

\section{Methodology}

3.1. Conceptual Framework. In the past twenty years between 2000 and 2020, many mixed reality systems supported many usage paradigms such as education, military, and robot. However, there were very few working on games using the feature of geo-based mixed reality to interactively support the story of the game and allow players to explore the game in a pioneering way. To the best of our knowledge, 


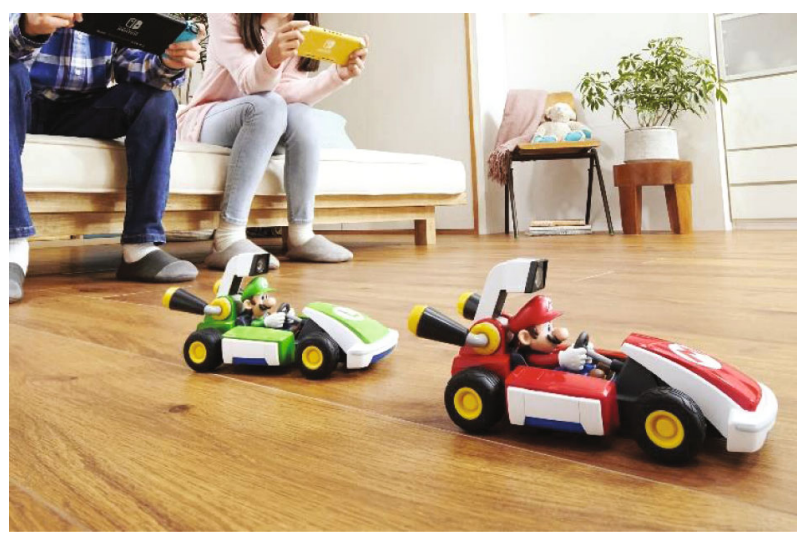

Figure 5: AR Mario Kart Live: Home Circuit from Nintendo using a classical concept of collective Mario Kart with the remotecontrolled car and mixed reality technology [12].

we find only five major games using the feature of geo-based mixed reality supporting the story of the game, which have tremendously impacted the mixed reality field in terms of either research or commercial purposes. In this way, Human Pacman, AR Car Game, Ingress, Pokémon Go, and AR Mario Kart Live are selected in performing market analysis and trend forecasting in this research study. Some other games are using the feature of geo-based mixed reality to support the story of the game, such as Harry Potter: Wizards Unite created by Niantic, the same gaming company building Ingress and Pokémon Go. Because this gaming company received major success with Pokémon Go in 2016, it later launched this Harry Potter-themed game in 2019. But the gaming concept of geo-based mixed reality is not so different from the previous games from the same company. Also, in terms of popularity, Pokémon Go is much more successful. Thus, we do not include the Harry Potter-themed game in this research. For this reason, we use only the five pioneering games (Human Pacman, AR Car Game, Ingress, Pokémon Go, and AR Mario Kart Live) for representing all the games of this kind in the past twenty years. Therefore, Human Pacman, AR Car Game, Ingress, Pokémon Go, and AR Mario Kart Live described in Section 2 are selected in performing market analysis and trend forecasting in this research study.

In this study, we use semiqualitative and exploratory methods to discover the relationships between the independent variable (year built) and the dependent variables (number of academic paper downloads, Google Trends, revenues, and number of downloads). The reason that we use year built as the independent variable is that it clearly helps us to gradually understand the evolution of technology from the early developmental stage to the growing stage, until the recent stage of geo-based mixed reality gaming. When performing market analysis, the first and most important task is to accurately identify target users/customers as different users/customers will lead to different expectations, methods, and market indicators. Speaking of geo-based mixed reality gaming, it is an emerging gaming style whose target users/customers still have high dynamics and are subject to significant changes according to trigger events happening each year. For example, in 2003, the target of geo- based mixed reality games is purely on researchers, so that the numbers of academic paper downloads and Google Trends are reasonable indicators for analyzing the academic market. Recently, as the popularity of geo-based mixed reality games has expanded to common gamers, additional indicators of game company revenues and the number of game downloads are added to cover another side of the market beyond academia. The significant effect of year built in market analysis will become very obvious in the next year as the introduction of Metaverse in October 2021 from Meta has hugely triggered the whole world, so that the future market of mixed reality will not be limited to academic persons and gamers but also architects, designers, investors, lawyers, and so on.

As explained earlier, in the past, target users/customers may mainly be researchers, and therefore, interpreting market success may require research indicators, such as academic paper download and Google Trends. However, in some certain years recently, target users/customers have evolved and expanded, such as nonresearchers and/or ordinary people. Thus, the market success indicator must be interpreted with other indicators such as revenues and the number of game downloads. It can be observed that the field of geo-based mixed reality gaming is quite new, compared relatively to some very old fields of science. Therefore, there is a wide variety of target audiences depending on the major trigger events in each year. Year built is then an important variable that will affect the dependent variables. These dependent variables are selected as our indirect indicators to reflect market and research trends regarding geo-based mixed reality games during the first two decades of the twenty-first century. However, the popularity of geo-based mixed reality games for academic research and commercial purposes is different in many aspects, and before Niantic's Ingress in 2013, there were only geo-based mixed reality games for academic research purposes. This means that for the games introduced with academic research purposes, they do not aim for or have no indicator regarding revenues and number of game downloads. Hence, during the years 2000 to 2013 , we portray the relationships between the independent variable and the dependent variables without revenues and number of game downloads. Figure 6 shows our conceptual framework before Niantic's Ingress was built. This conceptual framework emphasizes the essence and importance of the year built as the independent variable, highlighting periods in which geo-based mixed reality games are created. This independent variable is observed in conjunction with the dependent variables that include the number of academic paper downloads and Google Trends.

During the years 2013 to 2020, geo-based mixed reality games for commercial purposes have been included. Therefore, we redesign our conceptual framework as illustrated in Figure 7 . The independent variable is still the same which is year built. This independent variable can be seen to affect the dependent variables. However, the dependent variables do not only include the number of academic paper downloads and Google Trends but also include revenues and number of game downloads. This is to appropriately reflect the commercial purpose of these games. 


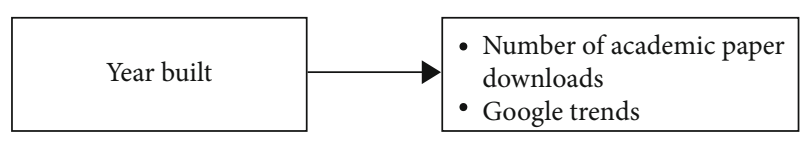

Figure 6: Our conceptual framework before Niantic's Ingress was built.

3.2. Data Collection Process. To begin analyzing the trends of geo-based mixed reality games, we collect data of mixed reality academic papers for the first two decades of the twentyfirst century from the IEEE Xplore digital library, one of the largest collections of research databases for academic papers and technical standards on computer science, electrical engineering, electronics, and related sciences. We use the keyword "Mixed Reality" to search the number of document titles found in the IEEE Xplore digital library in each year to understand the trends of academic papers for mixed reality. Figure 8 illustrates the gradual increase in the number of academic documents for mixed reality between 2000 and 2020. The vertical axis shows the number of documents entitled "Mixed Reality" as found in the IEEE database, while the horizontal axis shows the period of the research study. There is a small upward trend in the number of papers found between 2000 and 2002. Then, it does not increase until Human Pacman was broadcast on CNN and Discovery Channel in 2003. After that, it slightly keeps the increasing trend for mixed reality between 2004 and 2010 during the time that our paper on AR Car Game was internationally published. However, there is a small downward trend in the number of papers between 2011 and 2013. A possible reason is that these years were the starting era of massive smartphone usage and App Store was just launched a few years, so no smartphone and smartphone application were capable of the geo-based mixed reality games yet whereas there were many interesting geo-based mixed reality games on personal computers and laptops for about ten years (2003-2013). Nevertheless, the graph starts to increase again after 2013 when Ingress was launched. A possible reason is that Ingress gains popularity from its freemium business model, although it is built for commercial purposes. After Pokémon Go was released worldwide in 2016, there was a sharp and steep increase in academic paper trends for "Mixed Reality" between 2016 and 2018. It is obvious that, even though Pokémon Go focuses on the commercial aspect, it helps increase the popularity in academic research purpose for mixed reality as well. Finally, the number of document titles found was highest in 2020, when AR Mario Kart Live was released. In the future, we believe that the number of academic papers for mixed reality will be markedly increased, when a new game with new features and experiences is released. At the same time, to implement a new game, it needs an innovative method and algorithm from the academic community to solve the challenges of the technology.

Next, to analyze the trends of geo-based mixed reality games, we collect data of mixed reality from Google Trends for the first two decades of the twenty-first century. The data are analyzed from the popularity of top search queries in Google Search which is the highest worldwide market share (about 92\%) among all available search engines. Figure 9 depicts Google Trends worldwide for the "Mixed Reality" keyword between 2000 and 2020. We use the keyword "Mixed Reality" to collect the data from Google Trends in each year. The vertical axis shows the number of search interests of "Mixed Reality" in Google Search, while the horizontal axis illustrates the period of the research study. However, there is no data available in Google Trends before the year 2004, in which Human Pacman was approximately presented. There is a slight downward trend in the number of search interests found between 2004 and 2013. This means that the research works for geo-based mixed reality games (both Human Pacman and AR Car Game) do not significantly contribute to the popularity of mixed reality in Google Trends. A possible reason is partly about the incapacity of smartphones and built-in digital camera quality during these nine years. Since Ingress was internationally released, there is a slight upward trend for mixed reality from 2013 to 2016. After Pokémon Go was successfully debuted in 2016, there was an extremely steep increase in the number of search interests-about eight times higher in 2018 compared to 2016. The number of search interests was highest in 2018. Between 2018 and 2020, there is a downward trend until AR Mario Kart Live was debuted. A possible reason is that the trend already reached its peak during 2016-2018; after that, it was about time for the trend to saturate and find its appropriate and sustainable position in the long run unless any new trigger emerges. However, the number of search interests in 2020 is still more than five times compared to the number of search interests in 2016.

\section{Market Analysis of Geo-Based Mixed Reality Games}

This section performs the market analysis regarding geobased mixed reality gaming based on our five selected games (Human Pacman, AR Car Game, Ingress, Pokémon Go, and AR Mario Kart Live) from the previous section. Table 1 shows our conclusion regarding interesting aspects of the five selected geo-based mixed reality games. This table also includes dependent variables that cannot be illustrated as graphs due to insufficient public data like revenues and the number of game downloads. In detail, we will comparatively analyze each game based on the year built, the devices used in the game, the main purposes of the game, the number of academic paper downloads, the game revenues, the number of game downloads, and the types of virtual items in the game.

Mäyrä and Alha [13] gave a good overview for early history and development of mobile games. As stated before, Human Pacman, AR Car Game, Ingress, Pokémon Go, and AR Mario Kart Live are proposed in 2004, 2010, 2013, 2016 , and 2020, respectively. In the early years of the twenty-first century, geolocation mixed reality games often did not rely on their geo-based features on a smartphone; this was the case with Human Pacman and AR Car Game. In a detailed manner, Human Pacman uses a networked wearable computer with a head-mounted display (HMD) and mobile sensor, while AR Car Game uses a personal 


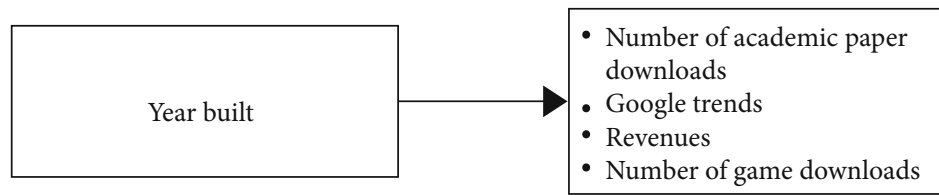

FIGURE 7: Our conceptual framework after Niantic's Ingress was created in 2013.

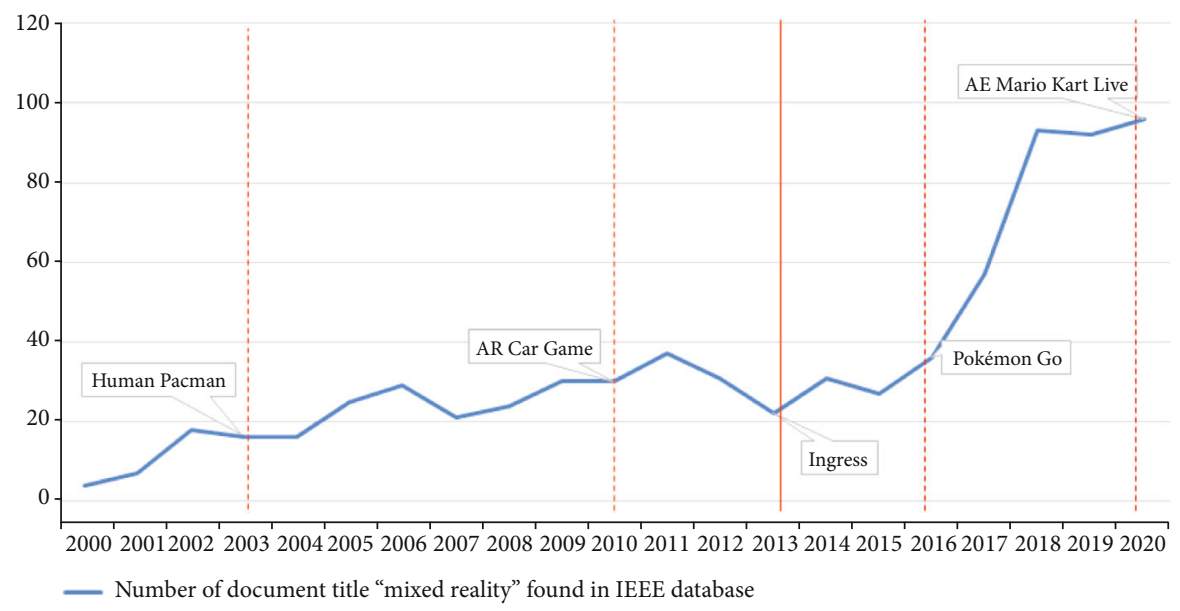

FIGURE 8: Academic paper trends for the "Mixed Reality" search keyword according to IEEE Xplore digital library for the past twenty years, which were collected on 25 September 2021.

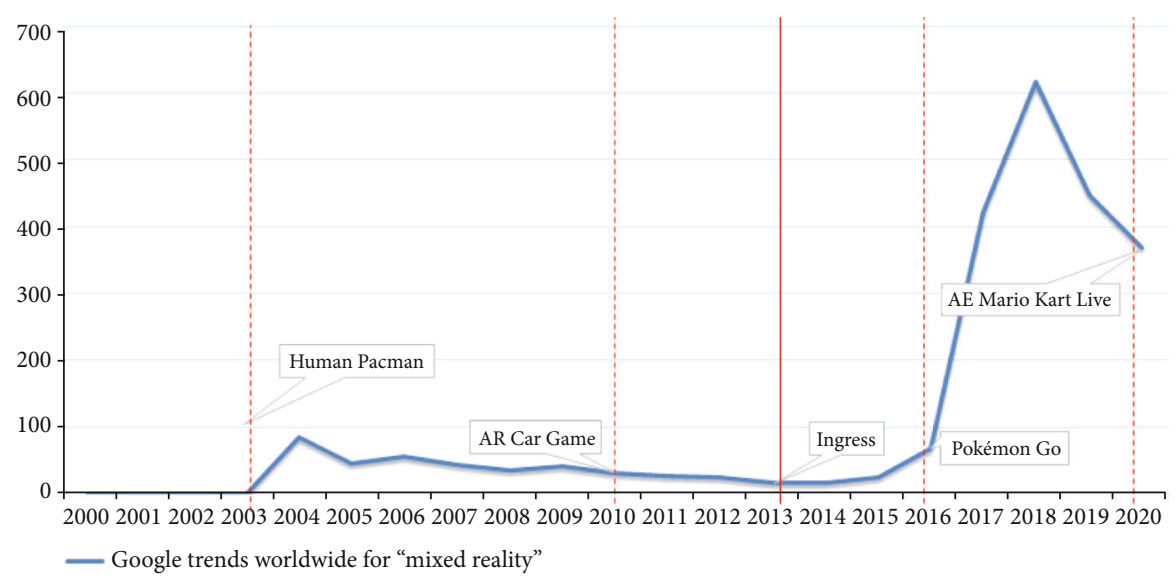

Figure 9: Google Trends worldwide for the "Mixed Reality" keyword in the past twenty years, collected on 25 September 2021.

computer or a laptop for running and playing games. This is because at that time, there was not enough suitable and capable smartphone that was suitable for creating geolocation mixed reality games. For instance, it was not possible to build the games on the App Store by Apple in the year 2000s because the App Store was initially opened in 2008. However, although the App Store started with a small number of applications available, it has opened the eyes to the possibilities for developing geolocation mixed reality applications on smartphones. This is because of the new capacities at a more affordable price regarding the computing power, cellular networks, internet connection, touchscreenbased user experiences and interfaces, resolution screen, and memory on the smartphone. For example, after iPhone 5 was first launched in 2012, we have seen more geolocation mixed reality games on smartphones available in the market, such as Ingress which was released in 2013 and later for iOS devices in 2014. Since then, the devices used in geo-based mixed reality games have gradually changed from laptops and personal computers to a smartphone. For example, Ingress and Pokémon Go use smartphones to play games. The only exception is AR Mario Kart Live that is played on a nonsmartphone mobile game console named the Nintendo Switch. Among the massive trend of smartphone gaming, this choice of Nintendo is understandable as it used to be a traditional Nintendo way to create their uniquely special game console in both hardware and software. In conclusion, we think that the choice of devices is partly related to the increasing market trend and popularity of geo-based mixed reality games as it directly reflects the number of 
TABLE 1: Comparison among five significant geo-based mixed reality games.

\begin{tabular}{|c|c|c|c|c|c|}
\hline Name & Human Pacman & AR Car Game & Ingress & Pokémon Go & AR Mario Kart Live \\
\hline Year built & 2004 & 2010 & 2013 & 2016 & 2020 \\
\hline Devices & $\begin{array}{l}\text { Wearable } \\
\text { computer and } \\
\text { HMD }\end{array}$ & $\begin{array}{l}\text { Laptop or } \\
\text { personal } \\
\text { computer }\end{array}$ & Smartphone & Smartphone & Nintendo Switch \\
\hline Main purposes & $\begin{array}{l}\text { Academic } \\
\text { research and } \\
\text { development }\end{array}$ & $\begin{array}{l}\text { Academic } \\
\text { research and } \\
\text { development }\end{array}$ & $\begin{array}{l}\text { Commercial but } \\
\text { freemium }\end{array}$ & $\begin{array}{l}\text { Commercial but } \\
\text { freemium }\end{array}$ & Commercial purpose \\
\hline $\begin{array}{l}\text { Number of } \\
\text { academic paper } \\
\text { downloads }\end{array}$ & $\begin{array}{l}344 \text { downloads } \\
\text { from ACM } \\
\text { database }\end{array}$ & $\begin{array}{l}297 \text { downloads } \\
\text { from IEEE } \\
\text { database }\end{array}$ & $\begin{array}{l}\text { Not available } \\
\text { (commercial } \\
\text { purpose) }\end{array}$ & $\begin{array}{c}\text { Not available } \\
\text { (commercial purpose) }\end{array}$ & $\begin{array}{l}\text { Not available (commercial } \\
\text { purpose) }\end{array}$ \\
\hline Revenues & No commercial & No commercial & $\begin{array}{l}20 \text { thousand USD in } \\
1 \text { month }(07.2019)\end{array}$ & $\begin{array}{l}5 \text { billion USD in } 5 \\
\text { years }(2016-2021)\end{array}$ & $\begin{array}{c}\text { Not yet released (though } 200 \\
\text { million USD for Mario Kart } \\
\text { Tour) }\end{array}$ \\
\hline $\begin{array}{l}\text { Number of game } \\
\text { downloads }\end{array}$ & $\begin{array}{l}\text { No commercial } \\
\text { download } \\
\text { allowed }\end{array}$ & $\begin{array}{l}\text { No commercial } \\
\text { download } \\
\text { allowed }\end{array}$ & $\begin{array}{c}20 \text { million downloads } \\
\text { in } 5 \text { years }(2013- \\
2018)\end{array}$ & $\begin{array}{c}632 \text { million } \\
\text { downloads in } 5 \text { years } \\
(2016-2021)\end{array}$ & $\begin{array}{l}1.27 \text { million copies in } 6 \text { months } \\
(10.2020-04-2021)\end{array}$ \\
\hline Virtual items & Yellow balls & $\begin{array}{l}\text { Mushrooms and } \\
\text { coins }\end{array}$ & Portals & Pokémons & Mushrooms and coins \\
\hline
\end{tabular}

potential users possessing the game-ready device. The bigger the number of potential users, the better chance of the game to gain more popularity.

In addition, at the beginning of the twentieth century, the main purposes of geolocation mixed reality games are divided into two main purposes: academic research and commercial. The purposes of Human Pacman and AR Car Game are mainly on research and development (R\&D) in university laboratories. Later, although Ingress and Pokémon Go are aimed at focusing on commercials, they mainly use a freemium business model-a mixing of the words "free" and "premium"-with some in-application purchases for extra in-game items. In detail, Ingress allows players to partly spend money for their locations to be used as portals, and then, it can make their stores a pilgrimage place for players in the game. Similarly, Pokémon Go is free to play, but its business model is combined with local advertising, in-app purchases for extra in-game items, and special event hosting (upon requested and sponsored). This means that for progressing the game faster or gaining a competitive advantage in the game, players may have to pay more money to enjoy the game. This strategy can translate into a realworld business, even though these two games still focus on the freemium business model. However, unlike the previous four mentioned geolocation mixed reality games, AR Mario Kart Live is aimed at the commercial aspect as players must purchase the AR Mario Kart Live: Home Circuit-Mario SetNintendo Switch Mario Set Edition to play the game. Although it is not very expensive, it costs about 75 USD for one set. In summary, we believe that the purpose of geolocation mixed reality games is partially linked to the increasing market trend and popularity of games, especially commercial purpose for it increases the number of people knowing this kind of field. This can be observed from the graph in Figure 9. By increasing the popularity, it later rein- forces the number of research papers in this area because it can make the research works more profitable.

Moreover, as the aims of Human Pacman and AR Car Game are mainly about research and development in university laboratories, there is no revenue data available publicly. For Niantic Inc.'s Ingress, according to [6], the approximately generated revenue in July 2019 is 20,000 USD for one month. Their revenue has exponentially increased when Niantic Inc. launched Pokémon Go which is a game sharing the same location data as Ingress. According to [14], the game revenue of Pokémon Go has reached five billion USD over the five years during 2016-2021, averaging one billion USD per year. Even though the world has faced the COVID-19 pandemic since 2020, this Pokémon Go game made 1.2 billion USD in revenue in 2020 which is a $41 \%$ increase from 2019. This is probably because the game has adapted properly during the outbreak [15] and allowed players to suitably play indoors, stay safely in place, and keep healthy [16] while playing, so that lockdown measures may even turn positive for the game and encourage more spending for Pokémon Go from the players [11]. Although Pokémon is originated in Japan, the United States is the highest revenue nation for Pokémon Go, accounting for $38 \%$ of the total revenue within five years (1.9 billion USD of 5 billion USD). For AR Mario Kart Live, as it has been launched for less than a year, Nintendo has not yet released the revenue for this specific game. However, though it may not be compared directly, [17] indicated that Nintendo's Mario Kart Tour has reached 200 million USD in a player spending in lifetime revenue. Conclusively, in our opinion, the revenue is partly connected to the increasing market trend and popularity of geo-based mixed reality games. This is since it attracts many software companies which are currently working in similar areas, such as Metaverse and internet of things (IoT), to build more geo-based mixed reality games. 
In terms of the number of game downloads, Human Pacman and AR Car Game are not designed for commercial download as they focus on research and development. Therefore, they are not comparable in this particular dependent variable. For the two games of Niantic, [18] indicated that Ingress has about 20 million downloads in five years during 2013-2018, while Pokémon Go has 632 million downloads in five years during 2016-2021 which is very impressive. In the total 632 million downloads of Pokémon Go, $77 \%$ of installation is from Google Play, while the Apple App Store accounts for $23 \%$. The highest country (18\% of the total downloads) is the United States with 115.5 million downloads, while Brazil and India are second and third, respectively. For AR Mario Kart Live: Home Circuit, [12] explained that this game has 1.27 million copies in about six months from October 2020 to April 2021. Overall, in our perspective, the number of game downloads is moderately linked to the growing market trend and popularity of geo-based mixed reality games. This is because it straightly reflects the number of possible game players. The larger the number of possible game players, the brighter opportunity of the game to obtain more popularity.

In terms of virtual items in the games, each game has different virtual items, except for AR Car Game and Mario Kart Live: Home Circuit that shares similar virtual items of mushrooms and coins for entertaining the players in a classical concept of collective Mario Kart with a remote-controlled car. For the other games, Human Pacman uses yellow balls to familiarize players with a traditional concept from PacMan, a maze chase action game from Namco in 1979. For Ingress, portals are virtually merged at real places in realworld map data [19], while hundreds of fictional species of collectible monsters from Pokémons are randomly mixed as virtual items in the game of Pokémon Go. In conclusion, in our view, the type of virtual items in the games is not related to the escalating market trend and popularity of geo-based mixed reality games in a straight manner. This is because although there are two games (AR Car Game and AR Mario Kart Live) that share the same type of virtual items, it can be seen from the graph in Figure 9 that it does not directly affect the trends in each period.

\section{Conclusion and Recommendations}

This paper has presented a summary of pioneering geobased mixed reality games during the first two decades of the twenty-first century. Five intensively pioneering games are selected for analysis based on the periods built as follows: Human Pacman, AR Car Game, Ingress, Pokémon Go, and AR Mario Kart Live. In this paper, we first discuss the technique behind every game, respectively. Then, we explain the methodology we used to assess the relationships between the independent variable and the dependent variables. After that, the geo-based mixed reality gaming market analysis of each game is conducted and extensively compared, in terms of the year built, the devices used in the game, the game purposes, the number of academic paper downloads, the game revenues, the number of game downloads, and the types of virtual items in the game.
Previously, a global positioning system was mainly utilized to implement geo-based mixed reality games in the early twentieth century. However, more recently, computer vision, deep learning, and big data analytics are great tools for estimating the poses and giving geolocation data to process in the games accurately and quickly [20]. Therefore, for achieving the advancement of these tools, the purpose of geolocation mixed reality games for research should be first encouraged to allow a period of trial and error, even though the research purpose of gaming may not directly and significantly relate to the increasing market trend and popularity of games, in the long run, comparing to the commercial purpose gaming, according to our investigation.

According to our study and projection, we believe that there are three significant factors to consider for achieving the geo-based mixed reality games in the 2020s, particularly for the research purpose. The three factors are the machine capacity, the quality and quantity of data, and the advances in computational algorithms. First, sufficient machine capacity is important to run and process the geo-based mixed reality games, especially the processing speed of the machine to make it possible to run an interactive mixed reality response in real time. This is evidently confirmed from our investigation as the emergence of the high-capacity smartphone is the important trigger that has led to the surge of geo-based mixed reality gaming trends. In the 2020s, we predict that more than $90 \%$ of new geo-based mixed reality games will be conducted in real time or almost real time. Rather than just smartphones alone, the devices used in the games will be more varied, including the internet of things, such as Facebook's Ray-Ban Stories [21] and Xiaomi's Smart Glasses [22]. Second, the quality and quantity of data are critically essential, including the robustness of data required for the state of being extrapolatable or learnable. Third, the advance in computational algorithms is very important to build smarter, faster, and more reliable geobased mixed reality games. Highly related fields for empowering geo-based mixed reality games are mostly in computer science and artificial intelligence fields, such as artificial neural networks, deep machine learning, sensor fusion, and realtime signal analysis. Like other recently popular artificial intelligent games and applications, there is the science of machine learning and deep learning lying underneath the recent geo-based mixed reality games like Pokémon Go and AR Mario Kart Live. To train good machine learning or deep learning applications or games, the big amount of data with great quality is crucial so any company or any nation that can take control or possess the power of data will also take the lead in the advancement of artificial intelligence, including geo-based mixed reality gaming. We believe that the fight for data ownership in terms of the strategic competition between nations and/or the new competition between technology companies will be heatedly seen undeniably in the 2020s.

According to our observation, the research purpose gaming and the commercial purpose gaming are reinforcing each other, and both contribute to the marketing trend and popularity at last. Hence, although the three factors mentioned earlier seem to focus on the research aspect, it will 
in turn commercially reinforce the game revenue of geolocation mixed reality games, the number of game downloads, and the choice of devices for playing games. Once technology readiness regarding the three factors is ready, we forecast that the overall market size of geo-based mixed reality games around the globe is expected to significantly escalate by the late 2020s. This forecast is particularly when the high-capacity devices are more affordable for most users as well as the $6 \mathrm{G}$ internet is fully available worldwide and allows artificial intelligence-based sensor data streaming and processing to be done in a fast and reliable manner.

\section{Data Availability}

No data were used to support this study.

\section{Conflicts of Interest}

The author declares that there are no conflicts of interest.

\section{Acknowledgments}

This research presented herein was partially supported by a research grant from the Research Center, NIDA (National Institute of Development Administration), Bangkok, Thailand.

\section{References}

[1] D. Vargo, L. Zhu, B. Benwell, and Z. Yan, "Digital technology use during COVID-19 pandemic: a rapid review," Human Behavior and Emerging Technologies, vol. 3, p. 642, 2020.

[2] K. Alha, E. Koskinen, J. Paavilainen, and J. Hamari, "Why do people play location-based augmented reality games: A study on Pokemon GO," Computers in Human Behavior, vol. 93, pp. 114-122, 2019.

[3] C. Kerdvibulvech, "Location-based augmented reality games through immersive experiences," in Augmented Cognition. HCII. Lecture Notes in Computer Science, 12776, D. D. Schmorrow and C. M. Fidopiastis, Eds., pp. 452-461, Springer, Cham, Switzerland, 2021.

[4] A. D. Cheok, K. H. Goh, W. Liu et al., "Human Pacman: a mobile wide-area entertainment system based on physical, social, and ubiquitous computing," Advances in Computer Entertainment Technology, vol. 8, pp. 360-361, 2004.

[5] C. Kerdvibulvech, "Real-time augmented reality application using color analysis," in IEEE Southwest Symposium on Image Analysis \& Interpretation (SSIAI), 2010, pp. 29-32, Austin, TX, USA, 2010.

[6] S. Laato, S. M. Hyrynsalmi, and M. Paloheimo, "Online multiplayer games for crowdsourcing the development of digital assets," in Software Business. ICSOB, S. Hyrynsalmi, M. Suoranta, A. Nguyen-Duc, P. Tyrväinen, and P. Abrahamsson, Eds., vol. 370 of Lecture Notes in Business Information Processing, pp. 387-401, Springer, Cham, Switzerland, 2019.

[7] H. Chen, R. Hsu, Y. Chen, W. Hsu, and P. Huang, "AR game traffic characterization: a case of Pokémon Go in a flash crowd event," in 19th Annual International Conference on Mobile Systems, Applications, and Services (MobiSys), pp. 493-494, Virtual Event Wisconsin, 2021.
[8] Y. Qin, “Attractiveness of game elements, presence, and enjoyment of mobile augmented reality games: The case of Pokemon Go," Telematics and Informatics, vol. 62, article 101620, 2021.

[9] D. D. Roure, J. A. Hendler, D. James, T. Nurmikko-Fuller, M. V. Kleek, and P. Willcox, "Towards a cyberphysical web science: a social machines perspective on Pokémon GO," in 10th ACM Conference on Web Science (WebSci), pp. 65-69, Boston Massachusetts, USA, 2019.

[10] S. Laato and S. Rauti, "Central themes of the Pokémon franchise and why they appeal to humans," in Hawaii International Conference on System Sciences (HICSS), pp. 1-10, Kauai, Hawaii, USA, 2021.

[11] A. I. Wang, "Systematic literature review on health effects of playing Pokémon Go," Entertainment Computing, vol. 38, article 100411, 2021.

[12] T. Whitehead, Feature: Nintendo Had A Spectacular Year, And May Have Big Plans, NintendoLife, 2021, https://www .nintendolife.com/news/2021/05/feature_nintendo_had_a_ spectacular_year_and_may_have_big_plans.

[13] F. Mäyrä and K. Alha, "Mobile Gaming," in The Video Game Debate 2: Revisiting the Physical, Social, and Psychological Effects of Video Games, R. Kowert and T. Quandt, Eds., pp. 107-120, Routledge, New York, NY, USA, 2020.

[14] P. Tassi, "'Pokémon GO' has made $\$ 5$ billion in five years," Forbes Magazine, vol. 6, 2021.

[15] A. I. Wang and A. Skjervold, "Health and social impacts of playing Pokémon Go on various player groups," Entertainment Computing, vol. 39, article 100443, 2021.

[16] S. Laato, T. H. Laine, and A. K. M. N. Islam, "Location-based games and the COVID-19 pandemic: an analysis of responses from game developers and players," Multimodal Technologies and Interaction, vol. 4, no. 2, p. 29, 2020.

[17] C. Chapple, "Mario Kart Tour surpasses 200 million downloads and \$200 million in lifetime revenue," Sensor Tower, 2021, https://sensortower.com/blog/mario-kart-tour-200million-downloads.

[18] G. Kumparak, "Niantic overhauls ingress to make it more welcoming for new players," TechCrunch, 2018, https:// techcrunch.com/2018/11/05/what-is-ingress-prime/.

[19] J. H. Lee, S. A. Keating, and T. W. Windleharth, "Challenges in preserving augmented reality games: a case study of Ingress and Pokémon GO," in 14th International Conference on Digital Preservation (iPRES), Kyoto, Japan, 2017.

[20] T. D. Do, J. J. LaViola, and R. P. McMahan, "The effects of object shape, fidelity, color, and luminance on depth perception in handheld mobile augmented reality," in IEEE International Symposium on Mixed and Augmented Reality (ISMAR), pp. 64-72, Porto de Galinhas, Brazil, 2020.

[21] M. Zuckerberg, "Introducing Ray-Ban Stories: first-generation smart glasses," 2021, https://about.fb.com/news/2021/09/ introducing-ray-ban-stories-smart-glasses/.

[22] S. Byford, "Xiaomi shows off concept smart glasses with MicroLED display," The Verge, Augmented Reality, vol. 14, 2021. 